\title{
Primer Registro De Stenotarsus Subtilis Arrow (Coleóptera: Endomychidae), En Diapausa Sobre Trampa Malaise, En El Parque Nacional Camino De Cruces.
}

\section{First Record Of Stenotarsus Subtilis Arrow (Coleoptera: Endomychidae), In Diapause Over Malaise Trap, In Camino De Cruces National Park.}

\author{
José A. Rivera ${ }^{1}$ y Alonso Santos Murgas ${ }^{2}$
}

Universidad de Panamá, Panamá, Museo de Invertebrados G. B. Fairchild. Email:

jriveralorenzo@gmail.com 1 ORCID: https://orcid.org/0000-0001-8551-8965

santosmurgasa@gmail.com 2 ORCID: https://orcid.org/0000-0001-9339-486X

Stenotarsus subtilis Arrow (Coleóptera: Endomychidae) es un escarabajo de hongos (Endomychidae), en el cual se han observado adaptaciones etológicas interesantes frente a condiciones desfavorables (Nedved \& Windsor, 1994). La isla Barro Colorado (Panamá) en 1980, fue el primer sitio donde se observó agregaciones de individuos de esta especie en diapausa cerca de la base de una palmera de Oenocarpus mapora H. Karst. Los escarabajos se mantienen en diapausa por alrededor de 10 meses al año, seis de los cuales forman parte de la temporada lluviosa y cuatro a la temporada seca (Wolda \& Denlinger, 1984).

Aproximadamente hace 21 años Roubik \& Skelley, (2001) presenta una clave para once especies conocidas de Stenotarsus con registros publicados, que ocurren en Panamá: Stenotarsus cuprivestis Gorham 1890; S. cyanopterus Gorham 1890; S. globosus GuérinMéneville 1857; S. lemniscatus Gorham 1890; S. obtusus Gerstaecker 1858; S. ovalis Arrow 1920; S. panamanus Gorham 1890; S. purpuratus Gerstaecker 1858; S. subtilis Arrow 1920; S. validicornis Gerstaecker 1858 .

En el presente trabajo se registra por primera vez agregaciones de Stenotarsus subtilis Arrow (Coleóptera: Endomychidae) en estructuras artificiales (Trampas Malaise, modelo Townes modificadas) en el Parque Nacional Camino de Cruces, provincia de Panamá.

\section{Stenotarsus subtilis Arrow}

Stenotarsus subtilis Arrow 1920. Agregación: Más 20,000 individuos $\quad$ Sexo: 
Material examinado: Panamá, Provincia de Panamá, Parque Nacional Camino de Cruces, coordenadas N 0903'05.2' ' W 079³6'.0, 207 m, 3 dic 2020, col A. Santos, M. y J. Rivera, trampa Malaise, modelo Townes modificadas.

Distribución: Stenotarsus subtilis Arrow se ha registrado en el bosque tropical de tierras bajas del Monumento Natural Barro Colorado, Panamá. Las observaciones de diapausa en $S$. subtilis se han registrado en dos sitios, en la Isla Barro Colorado 1980 y la Península Gigante 1993 (Nedved, 1996).

Hábitat y ecología: En muchos de los aspectos del ciclo de vida de $S$. subtilis son poco conocidas y aun faltan más estudios para poder entender mejor su reproducción, su área de alimentación y los factores que con certeza desencadenan la diapausa de esta especie tropical. Este nuevo registro de Stenotarsus subtilis Arrow en el Parque Nacional Camino de Cruces a 207 msnm nos proporciona nueva información sobre su distribución y comportamiento.

Comentarios: Stenotarsus subtilis Arrow, los individuos de la primera agregación que se observó en Isla Barro Colorado y que fue estudiada desde 1980, fue clasificas como Stenotarsus rotundus Arrow (Nedved 1996; Nedved y Windsor 1994; Wolda y Denlinger 1984.); en 1996 el hallazgo de una segunda agregación en la Peninzula Gigante la clasifican como Stenotarsus "gigante" (Nedved 1996). (Roubik \& Skelley, 2001) consideraron estas dos especies como conespecíficas y mediante estudios genéticos de aloenzimas confirmaron que ambos escarabajos pertenecían a la misma especie. También compararon los escarabajos agregados con las especies en la literatura de Panamá, clasificando esta especie de escarabajo que presenta agregación y diapausa como Stenotarsus subtilis.
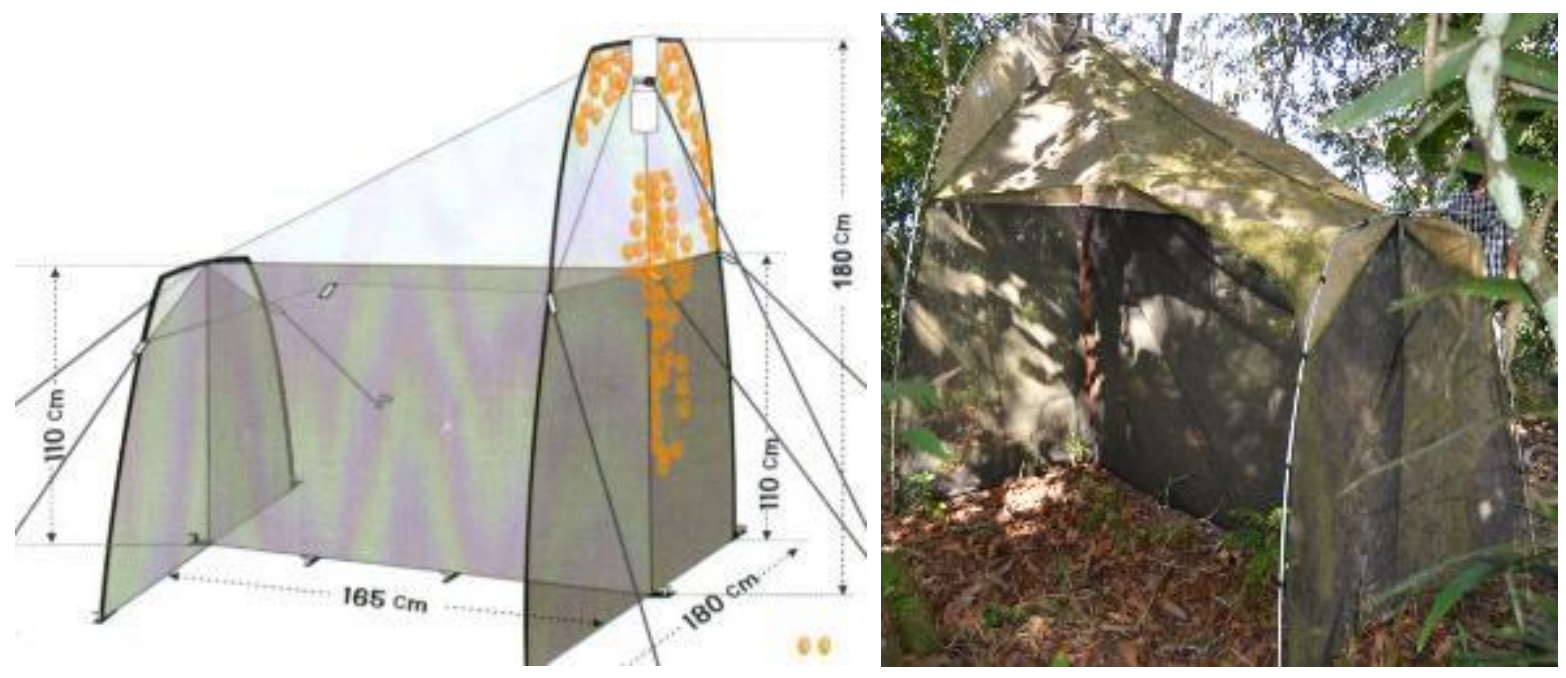

Figura 1. Zonas de agregación de S. subtilis en trampas Malaise, modelo Townes modificadas. 

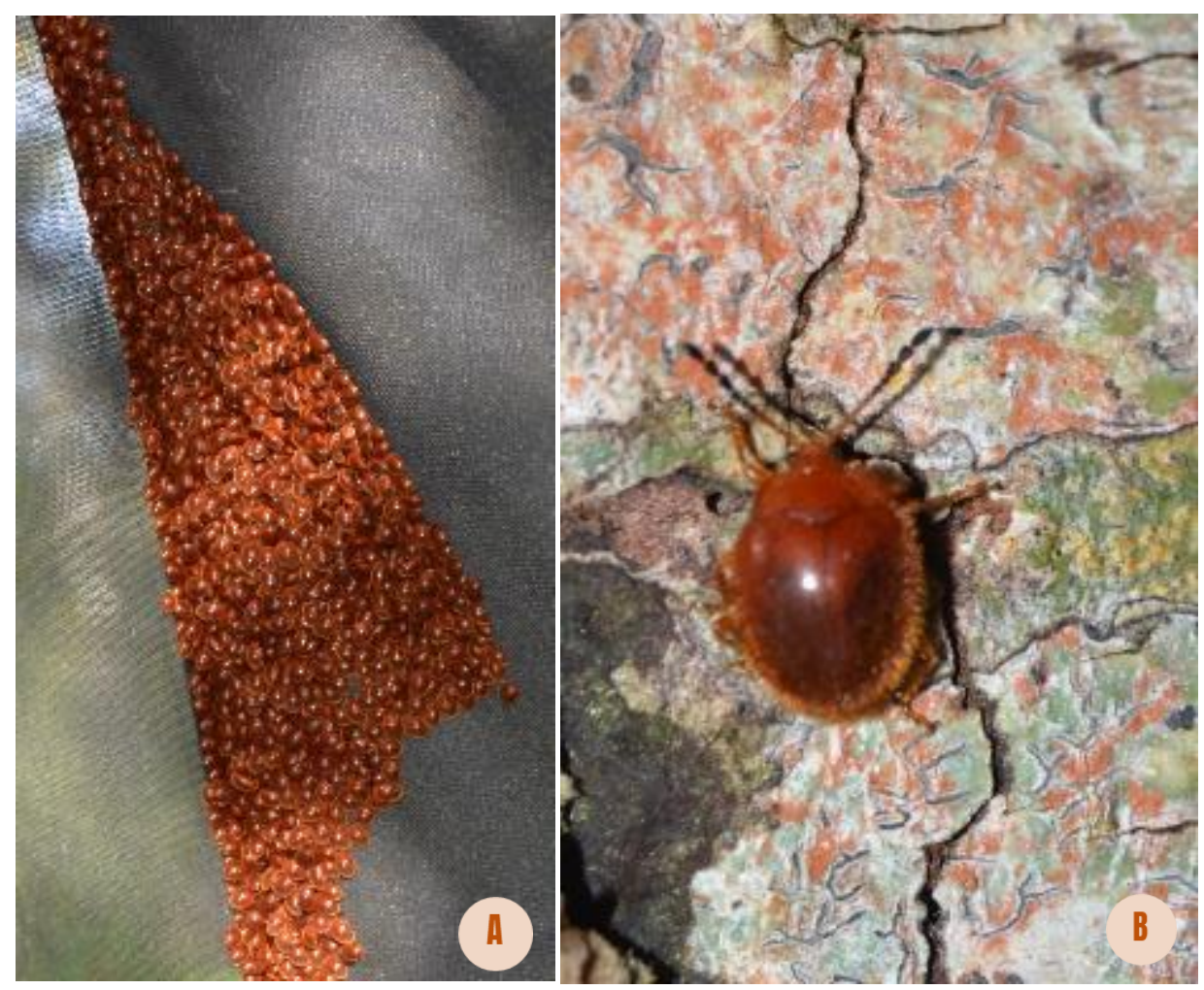

Figura 2. A) Stenotarsus subtilis en diapausa sobre Trampas Malaise. B) vita dorsal de Stenotarsus subtilis.

\section{REFERENCIAS BIBLIOGRÁFICAS}

NEDVED, O. (1996) "Beetle Tree The Second”, American Entomologist, 42(1), p. 3. doi: 10.1093/ae/42.1.3.

NEDVED, O. Y WINDSOR, D. (1994) "Supercooling ability, fat and water contents in a diapausing tropical beetle, Stenotarsus rotundus (Coleoptera:Endomychidae)", 
European Journal Entomology, pp. 307-312.

ROUBIK, D. W. Y SKELLEY, P. E. (2001) "Stenotarsus subtilis arrow, the aggregating fungus beetle of Barro Colorado Island nature monument, Panama (Coleoptera: Endomychidae)", Coleopterists Bulletin, 55(3), pp. 249-263. doi: 10.1649/0010065X(2001)055[0249:SSATAF]2.0.CO;2.

WOLDA, H. y DENLINGER, D. L. (1984) "Diapause in a large aggregation of a tropical beetle", Ecological Entomology, 9(2), pp. 217-230. doi: 10.1111/j.13652311.1984.tb00717.x. 